\title{
Ab Initio Characterization of Electron Transfer Coupling in Photoinduced Systems: Generalized Mulliken-Hush with Configuration-Interaction Singles
}

\author{
Hung-Cheng Chen ${ }^{\dagger}$ and Chao-Ping Hsu*, ${ }^{*,+}$ \\ Institute of Chemistry, Academia Sinica, 128 Section 2 Academia Road, Nankang, Taipei 115, Taiwan, and \\ Institute of Molecular Sciences, National Chiao-Tung University, 1001 Ta Hsueh Road, Hsinchu 300, Taiwan
}

Received: July 7, 2005; In Final Form: September 29, 2005

To calculate electronic couplings for photoinduced electron transfer (ET) reactions, we propose and test the use of ab initio quantum chemistry calculation for excited states with the generalized Mulliken-Hush (GMH) method. Configuration-interaction singles (CIS) is proposed to model the locally excited (LE) and chargetransfer (CT) states. When the CT state couples with other high lying LE states, affecting coupling values, the image charge approximation (ICA), as a simple solvent model, can lower the energy of the CT state and decouple the undesired high-lying local excitations. We found that coupling strength is weakly dependent on many details of the solvent model, indicating the validity of the Condon approximation. Therefore, a trustworthy value can be obtained via this CIS-GMH scheme, with ICA used as a tool to improve and monitor the quality of the results. Systems we tested included a series of rigid, $\sigma$-linked donor-bridge-acceptor compounds where "through-bond" coupling has been previously investigated, and a pair of molecules where "throughspace" coupling was experimentally demonstrated. The calculated results agree well with experimentally inferred values in the coupling magnitudes (for both systems studied) and in the exponential distance dependence (for the through-bond series). Our results indicate that this new scheme can properly account for ET coupling arising from both through-bond and through-space mechanisms.

\section{Introduction}

Electron transfer (ET) plays an important role in many chemical and biological processes in nature. ${ }^{1-4}$ For this reason, the ET reaction in organic and biological molecules has been the subject of continued experimental and theoretical research. Long-range intramolecular ET reaction has been under intensive study because of its direct relationship with the redox processes in many important biological systems. ${ }^{5-8}$ Electron donor (D) and acceptor (A) chromophores linked by a rigid, covalent spacer (bridge (B)), forming a D-B-A system, have attracted considerable attention because they allow systematic controls over molecular properties and fundamental characterization. Thereby, fundamental questions such as how the distance and orientation between donor and acceptor groups affect ET rates can be addressed experimentally. More importantly, D-B-A molecules are potential candidates for molecular devices, including molecular rectifiers, ${ }^{9,10}$ switches, ${ }^{11}$ wires,,${ }^{12}$ machines, ${ }^{13}$ photovoltaic cells, ${ }^{14,15}$ and nonlinear optical materials. ${ }^{16,17}$

For a nondiabatic ET reaction, the rate constant is generally described by ${ }^{1,18}$

$$
k_{\mathrm{ET}}=\frac{2 \pi}{\hbar}\left|V_{i f}\right|^{2}(\mathrm{FC})
$$

where $V_{i f}$ is the electronic coupling matrix element and $\mathrm{FC}$ is the Franck-Condon weighted density of states. Fundamental characterization of $V_{i f}$ is important for understanding the nature of ET reactions. For example, experimental studies have shown

\footnotetext{
* Corresponding author. Fax: 886-2-2783-1237. E-mail: cherri@ sinica.edu.tw.

Academia Sinica.

$\doteqdot$ National Chiao-Tung University.
}

that long-range ET couplings can be mediated by the throughbond and through-space mechanisms. ${ }^{19,20}$ Moreover, the distance dependence of ET coupling magnitudes is an important characteristic for bridge mediated couplings. For insulators, there exists an exponential distance dependence which is described as $^{21-23}$

$$
\left|V_{i f}\right|^{2}=\left|V_{i f}^{0}\right|^{2} \exp \left[-\beta\left(r-r_{0}\right)\right]
$$

where $V_{i f}^{0}$ is the electronic coupling element at a reference donor-acceptor distance $r_{0}$, and $\beta$ represents the rate of decay over distances. Experimental studies have suggested that $\beta$ is around $2.8-3.0 \AA^{-1}$ for through-space ET, ${ }^{24}$ about $0.8-1.1 \AA^{-1}$ for through-bond ET in saturated hydrocarbon bridges, and about $0.90 \AA^{-1}$ in rigidly fused cyclohexane..$^{25-27}$

For ET reactions in photoinduced systems, the initial state is a locally excited (LE) state and the final state is a charge-transfer (CT) state. The photoexcitation creats a LE state localized to either the donor or the acceptor fragment, and the subsequent charge-separation may be an ET or a hole-transfer (HT) event (see ref 28-30, for example) depending on the nature of the molecule. Without loss of generality, in this report, we will focus on systems where the donor is photoexcited, and an ET reaction was induced; i.e.,

$$
\mathrm{DBA} \stackrel{h v}{\longrightarrow} \mathrm{D} * \mathrm{BA} \rightarrow \mathrm{D}^{+} \mathrm{BA}^{-}
$$

To calculate ET coupling involving excited states, the generalized Mulliken-Hush $(\mathrm{GMH})^{31}$ has been a widely used method. The GMH method assumes that the dipole operator in the diabatic basis is diagonal, allowing values from the adiabatic space to be used to calculate the Hamiltonian off-diagonal matrix elements (in the diabatic space). We note that the zero transition 
dipole assumption restricts its application to transitions with a charge-transfer nature only. Unlike most energy-gap based methods, the application of GMH is not restricted to the transition state geometry, making GMH an attractive method for large systems. In GMH, the coupling strength is given as ${ }^{31}$

$$
V_{i f}=\frac{\mu_{i f} \Delta E_{i f}}{\sqrt{\Delta \mu_{i f}^{2}+4 \mu_{i f}^{2}}}
$$

where $\mu_{i f}$ is the transition moment, $\Delta E_{i f}$ is the energy difference, and $\Delta \mu_{i f}$ is the permanent dipole moment difference, all of which are calculated between the initial and final states of an ET reaction. Calculation of the electronic coupling value requires simultaneously characterizing the LE and CT states, typically through an excited-state calculation. In previous applications, ${ }^{32,33}$ semiempirical ZINDO/S has been used. ZINDO/S is known to reproduce spectroscopic properties well for organic molecules. ${ }^{34}$ Thus, the spectroscopic parameters derived from ZINDO/S Hamiltonian allow reasonable estimation of the ET coupling via the GMH scheme.

There are several ab initio schemes to calculate ET coupling between two charge-localizing ground states, such as direct coupling, ${ }^{35-37}$ Hartree-Fock Koopmans Theorem, ${ }^{38,39}$ or the recently developed spin-flip scheme. ${ }^{40}$ These first-principle methods are attractive because they allow systematic improvements and do not use empirical parameters. However, estimations of ET coupling involving excited states with ab initio methods have been rare. MCSCF wave functions were used in a block diagonalization scheme. ${ }^{41}$ One related work tested for the Condon approximation in ET coupling using CIS-GMH. ${ }^{42}$ For a biphenyl alcohol radical anion, a nonphysical change in the GMH coupling is reported as the torsion angle between the two phenyl groups varies. In this case, the two $\pi^{*}$ molecular orbitals are similar in their energies but have different electron occupation, a situation that often leads to a large nondynamical correlation effect, and this may contribute to the observed nonphysical behavior. In another recent report, time-dependent density functional theory (TDDFT) with GMH was used to calculate the ET coupling between the ground state and a series of excited states of a molecule. ${ }^{43}$ However, the validity of the most commonly used density functionals in calculating chargetransfer properties is still in question. ${ }^{44}$

An alternative approach is to calculate GMH coupling with ab initio wave function based theoretical models, which are not excluded from treating charge-transfer states. As the simplest ab initio model for excited state, CIS can be used for very large molecules. The energy of a CT state has been shown to exhibit correct Coulombic $1 / r$ distance dependence in state energies. ${ }^{44}$ Therefore, it is desirable to develop a CIS-based scheme for ET coupling through the GMH method.

Most of the ET reactions studied take place in a condensed phase, but previous calculations of ET coupling rarely treated the surrounding media. There are many solvent models that account for the influence of a dielectric medium in a quantum calculation (e.g., see ref 45 and references therein). As a simplification, point charges have been used to characterize the effect of amino acid residues when modeling a molecule embeded in a protein. ${ }^{46}$ It was shown that such simple approaches can yield good results for ET couplings in the reaction center protein of a photosynthetic purple bacterium. Point charge models offer the approximate long-range interaction with the environment, and they are computationally simple to implement. The image charge approximation (ICA) ${ }^{47}$ offers the first-order effect arising from the dielectric media, which is useful in molecular dynamics simulation in place of bulk dielectric. ${ }^{48}$ In the present work, we test to see if a simple ICA model could be useful in quantum chemical computation for ET couplings.

In this work, we propose to use ab initio quantum calculation to characterize the electronic coupling of photoinduced ET reactions, i.e., coupling of two excited states, a locally excited (LE) state and a charge-transfer (CT) state. We use CIS to characterize excited states and GMH to calculate ET coupling. We will show that the CT state is sometimes coupled to a highlying locally excited state, leading to a large transition dipole and consequently overestimating the ET coupling. To remove such an artifact, we propose employing a solvent model to lower the energy of the CT state. A simple model such as ICA reduces the energy of the CT state, and as a result, the erroneous mixing with the high energy LE states is removed. The coupling strength is largely insensitive to many details of the solvent model. We show that ICA improves the quality of the result without increasing the computational complexity and can be used to monitor the quality of ET couplings. The calculated GMH coupling strengths agree well with experimentally derived values.

\section{Theory and Methods}

2.1. Image Charge Approximation. We model the solvent reaction field using the image charges arising from the Mulliken atomic charges, which are derived from a CT state. We assume the solute molecule is placed in a spherical cavity embedded in a dielectric medium. The position and magnitude of such image charges were derived in ref 47 . The reaction potential $V_{\mathrm{im}}$ is approximated as the potential created by the image charges $q_{A}^{i m}$ at $\vec{r}_{A}^{i m}$ that are defined as

$$
q_{\mathrm{A}}^{i m}=-\frac{\epsilon-1}{\epsilon+1} \frac{\left|r_{\mathrm{A}}\right|}{R} q_{\mathrm{A}}
$$

located at

$$
\vec{r}_{\mathrm{A}}^{i m}=\frac{R^{2}}{\left|r_{\mathrm{A}}\right|^{2}} \vec{r}_{\mathrm{A}}
$$

where $R$ is the radius of the spherical cavity, $\epsilon$ is the dielectric constant of the medium, $\vec{r}_{\mathrm{A}}$ is the position vector of the atom $\mathrm{A}$, and $q_{\mathrm{A}}$ is the Mulliken charge on atom A. Unless noted otherwise, in all of our ICA models, the origin was at the center of nuclear charges of the molecule, and a cavity radius $R=$ $\left(r_{\max }+1.5\right) \AA$ was used, where $r_{\max }$ is the largest distance among all $r_{\mathrm{A}}$ 's.

In our calculation, the Mulliken charges $\left\{q_{\mathrm{A}}\right\}$ are calculated from the CT state. The charge distribution is changed when the solvent model is employed. Therefore, a self-consistent iteration should be used to obtain a converged solution. As will be shown in the following sections, the calculated ET coupling is weakly dependent on the details of the solvent model, including the use of the self-consistent iteration, and this weak dependency is consistent with the Condon approximation. ${ }^{49}$ Therefore, unless indicated otherwise, all of the results reported in this paper were obtained using the Mulliken charges derived in a vacuum.

2.2. Through-Bond vs Through-Space Coupling. In $D-B-A$ molecules, the overall ET coupling is composed of two contributions: ${ }^{50-52}$

$$
V_{i f}=T_{\mathrm{DA}} \equiv V_{\mathrm{DA}}^{0}+\sum_{B} \frac{V_{\mathrm{DB}} V_{\mathrm{BA}}}{E-E_{\mathrm{B}}}
$$




\section{CHART 1: Series of Norbornyl-Linked D-B-A Molecules $^{a}$}

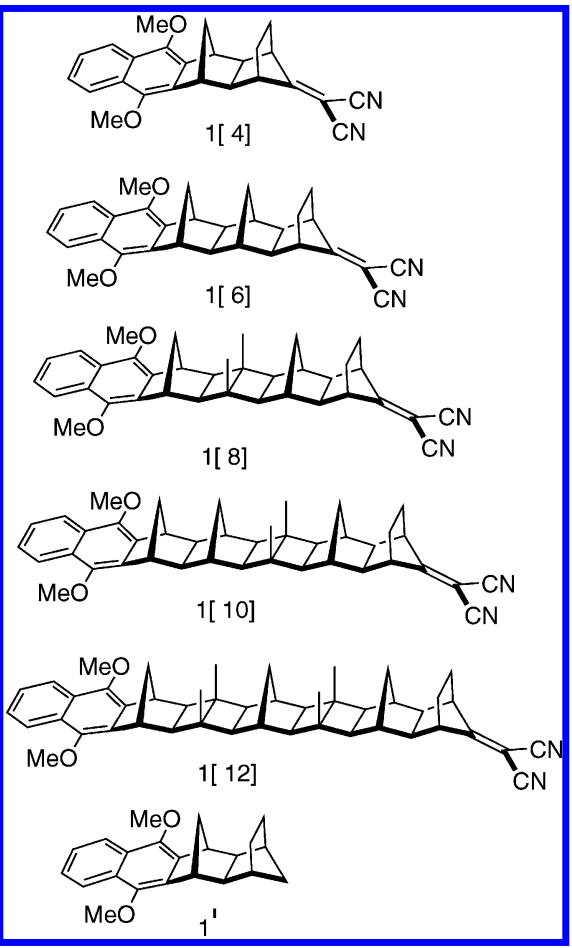

${ }^{a}$ In the square brackets are the number of $\sigma$ bonds in the linking spacers. $\mathbf{1}^{\prime}$ was used to find the LE state structure.

\section{CHART 2: Pair of Molecules Where Through-Space ET} Coupling Was Studied ${ }^{a}$

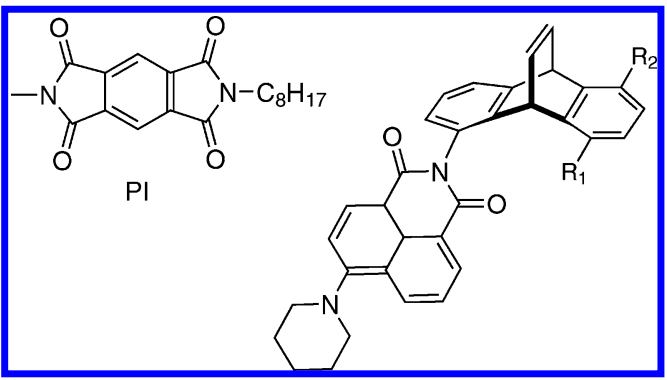

a 2a: $\mathrm{R}_{1}=\mathrm{PI}, \mathrm{R}_{2}=$ H. 2b: $\mathrm{R}_{1}=\mathrm{H}, \mathrm{R}_{2}=$ PI. A third molecule $\mathbf{2}^{\prime}$ was used to find the structure of the LE state, where $R_{1}=R_{2}=H$.

where $E$ is the energy of the electron being transferred. The first term $V_{\mathrm{DA}}^{0}$ represent the through-space contribution, and the second term is a bridge-mediated superexchange coupling, which is also known as the through-bond contribution.

We calculated $V_{\mathrm{DA}}^{0}$ using a model containing disconnected donor and acceptor fragments. Hydrogen atoms were added to fill broken covalent bonds. The positions of these extra $\mathrm{H}$ atoms were optimized to minimize the artifact of truncation. If the calculated $V_{\mathrm{DA}}^{0}$ was much less than that of the full molecule, we concluded that the second term, bridge-mediated coupling, was important. If, however, $V_{\mathrm{DA}}^{0}$ was close to the full-molecule coupling $V_{i f}$ calculated, we concluded that the through-space coupling was in effect.

2.3. Other Computational Details. For a systemic characterization of the CIS-GMH scheme, we calculated the electronic coupling in photoinduced ET reactions in the well-studied series of dimethoxynaphthalene (DMN) - [polynorbornyl- $(n, \sigma$-bonds)]dicyanovinyl (DCV) molecules, denoted as the $\mathbf{1}[\boldsymbol{n}]$ series (Chart 1) ${ }^{28}$ Previous studies of $\mathbf{1}[\boldsymbol{n}]$ have shown that ET is mainly through superexchange mediated by the $\sigma$ bonded spacers, with

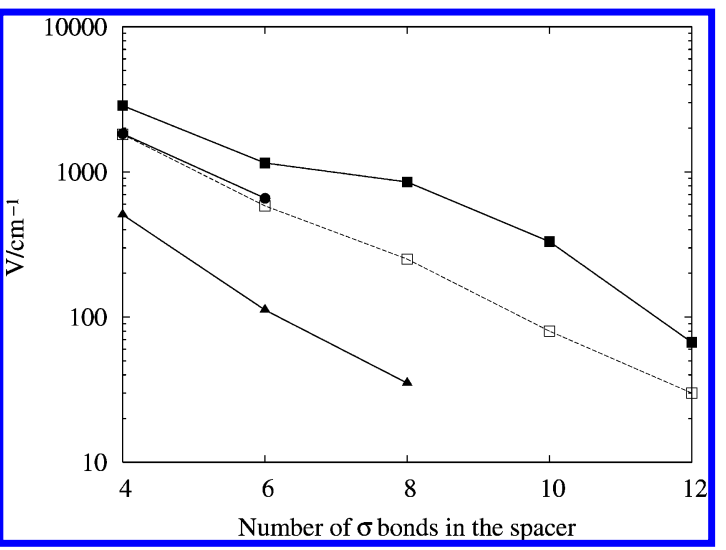

Figure 1. Calculated ET coupling strengths compared with experimental estimates and other previous results. Data with squares are CISGMH results with the $6-31 \mathrm{G}^{*}$ basis. Filled squares are calculated in a vacuum, and open squares are calculated using a solvent model with $\epsilon$ $=37.5$ (mimicking acetonitrile). Filled circles are values inferred from experimental results. ${ }^{58}$ Filled triangles represent results from a CNDO Hamiltonian. ${ }^{57}$

the rigid norbornylogous units holding the donor and acceptor fragments at a fixed distance and relative orientation. We further tested the same calculation scheme on a pair of molecules where 4-piperidinylnaphthalene-1,8-dicarboximide (ANI) was the electron donor, and $N$ - $(n$-octyl)pyromellitimide (PI) was the electron acceptor, and both were attached to either 1,5- or 1,8-positions of a dibenzobicyclo[2.2.2]octatriene (DBO) spacer, forming either a cofacial or noncofacial compound. The molecules were denoted as $\mathbf{2 a}$ and $\mathbf{2 b}$ shown in Chart 2. ${ }^{29}$ They have been reported as a system that demonstrates through-space ET coupling.

For all molecules calculated in this work, we started with ground state geometries that were optimized with the density functional theory (DFT) using B3LYP ${ }^{53} / 6-31 \mathrm{G}^{*}$ settings. Photoinduced ET reactions take place in the LE state where excitation is localized in the donor fragment. For an approximated structure of vibrationally relaxed LE state, we used a model molecule composed of a donor and a short bridge (e.g., molecules $\mathbf{1}^{\prime}$ and $\mathbf{2}^{\prime}$ ) and optimized it in its $S_{1}$ surface at the CIS/6-31G* level. The desired relaxed D*BA structure was obtained by assembling the model molecule with the rest of the full molecule in its ground-state structure. ${ }^{54}$ Because the LE state is insensitive to solvation, we have performed the optimization without the solvent model. We show that, for 1[8], the structure built this way and the GMH couplings were very close to those of the fully relaxed $S_{1}$ state (Tables $S_{1}$ and S2 in the Supporting Information). Their difference in $S_{1}$ state energy was less than $0.01 \mathrm{eV}$. Quantities $\mu_{i}, \Delta E_{i f}$, and $\Delta \mu_{i f}$ as required for evaluation with eq 4 were obtained from a CIS calculation. A developmental version of quantum chemistry package Q-Chem ${ }^{55}$ was used for all calculations.

\section{Results and Discussion}

For the $\mathbf{1}[n]$ series, we tested for the effects of many solvent details and compared our results with previously reported theoretical and experimental values. Results for molecules 2a and $\mathbf{2 b}$ are reported and discussed in section 3.4.

3.1. Effects of a Solvent Model. In Figure 1, results using GMH ( eq 4) from CIS calculations are reported. Without a solvent model, the coupling strengths for small bridges (1[4] and 1[6]) are within an acceptable range when compared to numbers derived from experimental results. However, in the semilog plot, the coupling strengths do not follow a straight 
TABLE 1: Electron Transfer Coupling $\left(\mathrm{cm}^{-1}\right)$ of the $1[n]$ Series

\begin{tabular}{|c|c|c|c|c|c|c|}
\hline \multirow[b]{2}{*}{ solvent } & \multirow[b]{2}{*}{ basis set } & \multicolumn{5}{|c|}{ bridge $\sigma$ bond numbers $(n)$} \\
\hline & & 4 & 6 & 8 & 10 & 12 \\
\hline \multirow[t]{2}{*}{ vacuum } & $3-21 G^{*}$ & 2393 & 1302 & 673 & 493 & 143 \\
\hline & $6-31 G^{*}$ & 2863 & 1153 & 852 & 331 & 67.3 \\
\hline \multirow[t]{2}{*}{ benzene } & $3-21 G^{*}$ & 1974 & 805 & 350 & 126 & 43.5 \\
\hline & $6-31 G^{*}$ & 1972 & 791 & 289 & 100 & 34.8 \\
\hline \multirow[t]{2}{*}{ acetonitrile } & $3-21 G^{*}$ & 1843 & 622 & 276 & 87.3 & 35.5 \\
\hline & 6-31G* & 1815 & 584 & 251 & 80.1 & 30.3 \\
\hline \multicolumn{2}{|l|}{$\mathrm{CNDO} / \mathrm{S} / \mathrm{CI}^{a}$} & 533 & 119 & $34^{b}$ & & \\
\hline \multirow{2}{*}{\multicolumn{2}{|c|}{$\begin{array}{l}\mathrm{CNDO} / \mathrm{S} / \mathrm{CI}^{c} \\
\text { experimentally inferred }^{d}\end{array}$}} & 507 & 112 & 35 & & \\
\hline & & 1830 & 660 & & & \\
\hline \multicolumn{7}{|c|}{ Without the Spacer: Disconnected Model } \\
\hline vacuum & $6-31 G^{*}$ & 396 & 0.678 & 0.00735 & $<0.007$ & $<0.007$ \\
\hline
\end{tabular}

TABLE 2: Major Excitations and Their Amplitudes (in Parentheses) of the First Excited State (LE) and the Lowest Charge-Transfer (CT) State for 1[6] in a Vacuum and in Two Solvents (Basis Set: 6-31G*)

\begin{tabular}{llll}
\hline \multicolumn{1}{c}{ vacuum } & \multicolumn{1}{c}{ benzene } & \multicolumn{1}{c}{ acetonitrile } \\
\hline $\mathrm{LE}$ & $\mathrm{HOMO} \rightarrow$ LUMO+1 $(0.90)^{a}$ & HOMO $\rightarrow$ LUMO+1 $(0.91)^{a}$ & HOMO $\rightarrow$ LUMO+1 $(0.91)^{a}$ \\
& HOMO-1 $\rightarrow$ LUMO+2 $(-0.34)^{a}$ & HOMO-1 $\rightarrow$ LUMO+2 $(-0.34)^{a}$ & HOMO-1 $\rightarrow$ LUMO+2 $(-0.34)^{a}$ \\
$E / \mathrm{eV}^{b}$ & 4.64 & 4.65 & 4.65 \\
$\mathrm{CT}$ & $\mathrm{HOMO} \rightarrow$ LUMO $(0.83)^{c}$ & HOMO $\rightarrow$ LUMO $(0.88)^{c}$ & HOMO $\rightarrow$ LUMO $(0.98)^{c}$ \\
& HOMO $\rightarrow$ LUMO+1 $(-0.27)^{a}$ & HOMO-2 $\rightarrow$ LUMO+1 $(-0.33)^{a}$ & 6.34 \\
$\theta^{d}$ & 7.26 & 6.91 & 6.53
\end{tabular}

${ }^{a}$ Excitation localized in donor. ${ }^{b}$ Vertical excitation energy. ${ }^{c}$ Charge-transfer excitation. ${ }^{d}$ Angle (in degrees) between $\Delta \mu_{i j}$ and $\mu_{i j}$, in a transition from the LE to the CT state.

line, an expected behavior for insulating spacers such as the $\sigma$-bonded fragments for electrons coming from a $\pi-\pi^{*}$ excitation. ${ }^{21-23,56}$

When a molecule is modeled in a vacuum, the CT state is high in energy. The validity of CIS solutions for high-lying states is limited, because double or higher excitation may play a more important role than it does in the low-energy region. In addition, the density of electronic states is much higher than the typical valence transition region. Thus, the CT state may be in the close vicinity of locally excited states. If that happens, the eigenstate from such a CIS calculation will be a mixture of CT and highenergy LE states. As a result, the transition moment becomes large due to that mixing, leading to a biased result.

Most photoinduced ET experiments are performed in solution, where the CT states are significantly stabilized. Thus, with a solvent model, we can lower the energy of CT states and possibly avoid the problems of using the high-energy state in CIS. We have used the ICA model described above (section 2.1) to account for the long-range electrostatic interaction between the molecule and its surrounding solvent. Results are presented in Figure 1 and Table 1. It is shown that the model solvent offers a significant improvement to the results. There is a smooth exponential decay as the spacer becomes longer. The magnitudes of coupling strengths are also much closer to the experimentally inferred values. In addition, with a solvent model, the coupling magnitudes become weakly dependent on the basis sets used; i.e., results from $3-21 \mathrm{G}^{*}$ and $6-31 \mathrm{G}^{*}$ basis sets are very similar. For comparison purposes, previous computational results with CINDO/S/CI ${ }^{57}$ are included in Figure 1. It is shown that ab initio methods offer larger coupling strengths compared to semiempirical models. As discussed below in section 3.3.1, the rate of exponential decay with distances is smaller for ab initio results than for semiempirical Hamiltonians.

In Table 2, we listed important configurations in CIS calculations. It can be seen that the mixing of local excitation in the CT state is suppressed with the solvent model, leading to a smaller transition dipole and a reduced coupling. The angles between the permanent dipole moment differences $\left(\Delta \vec{\mu}_{i j}\right)$ and the transition dipole moments $\left(\vec{\mu}_{i j}\right)$ are also listed. It is shown that, upon inclusion of a solvent, the angle between the two dipole vectors decreases. Therefore, the use of a solvent model increases the applicability of the GMH expression, where an assumption of $\Delta \vec{\mu}_{\mathrm{i} j}$ being parallel to $\vec{\mu}_{i j}$ was made.

3.2. Sensitivity to Details of the Solvent Model. 3.2.1. Solvent Polarities and Cavity Sizes. To see how the details of a solvent model may affect the results, we performed a series of tests over different solvent polarities and cavity sizes. Relevant quantities of GMH coupling were included in Figure 2. The figure shows that, with a solvent, the energy gap between the LE and CT states decreases almost linearly with solvent polarity. The energy of the LE state changes very little $(<0.02$ $\mathrm{eV}$ ) in this test (data not shown). This linear dependence of excitation energy on solvent polarity is characteristic of a chargeseparated state, and is correctly reproduced in the CIS calculation. The transition dipole moment between the LE and CT state $\left(\mu_{i f}\right)$ decreases, then increases. The initial decrease of $\mu_{i f}$ reflects the change in the composition of the CT state. As shown in Table 2, the locally excited configuration is greatly reduced when a model solvent is added.

As shown in Figure 2, in the high-polarity region (solvent polarity $\geq 0.3$ ), the transition dipole moment $\mu_{i f}$ increases as polarity increases, and the product $\Delta E_{i f} u_{i f}$, the numerator of the GMH expression (eq 4), remains nearly a constant. The final coupling value $V$ is also roughly a constant in this region. Similar trends are also observed when the cavity size is varied (right panel, Figure 2): the final coupling strength $V$ changes little when the radius of the sphere cavity is changed. Such a result is consistent with the Condon approximation, which states that electronic coupling is weakly dependent on most external degrees of freedom such as external fields or nuclear coordinates. ${ }^{49}$ 


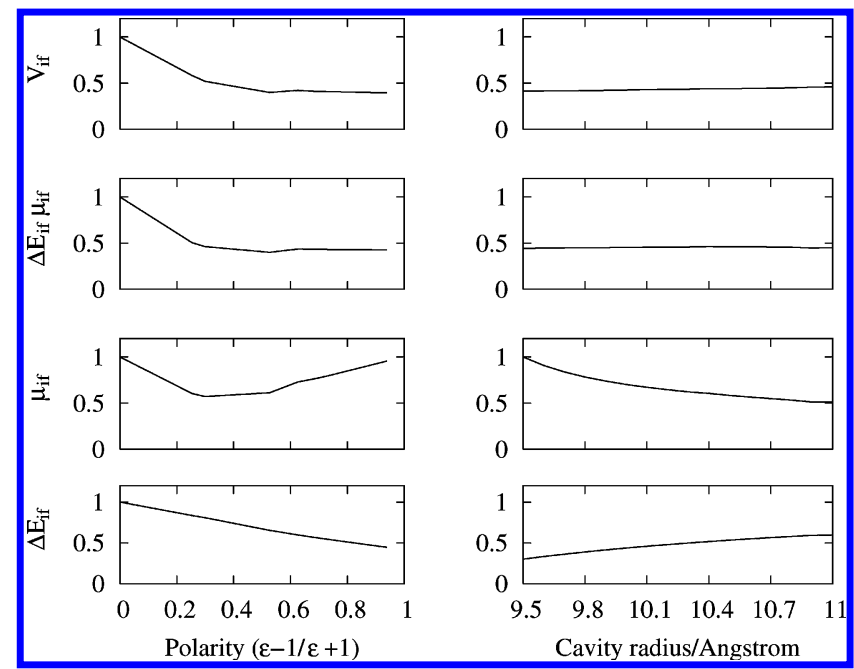

Figure 2. Effects of solvent polarity (left) and cavity radii (right) on quantities determining ET coupling of $\mathbf{1}[\mathbf{8}]$. The values are normalized to 1 for the maximum values in both panels. For testing solvent polarity, a cavity radius of $10.5 \AA$ was used. For the cavity radius data, the dielectric constant was fixed at 37.5.

\section{TABLE 3: Iterations toward Self-Consistency in ICA ${ }^{a}$}

\begin{tabular}{|c|c|c|c|c|c|c|c|c|}
\hline \multirow[b]{2}{*}{ cycle } & \multicolumn{4}{|c|}{ solvent: benzene $(\epsilon=2.28)$} & \multicolumn{4}{|c|}{ solvent: acetonitrile $(\epsilon=37.5)$} \\
\hline & $\begin{array}{l}\Delta E / \\
\mathrm{eV}^{b}\end{array}$ & $\begin{array}{l}\Delta \mu / \\
\mathrm{au}^{c}\end{array}$ & $\begin{array}{l}\mu_{i f}{ }^{d} \\
\mathrm{au}^{d}\end{array}$ & $\begin{array}{c}V / \\
\mathrm{cm}^{-1 e}\end{array}$ & $\begin{array}{l}\Delta E / \\
\mathrm{eV}^{b}\end{array}$ & $\begin{array}{l}\Delta \mu / \\
\mathrm{au}^{c}\end{array}$ & $\begin{array}{l}\mu_{i f}{ }^{\prime} \\
\mathrm{au}^{d}\end{array}$ & $\begin{array}{c}V / \\
\mathrm{cm}^{-1 e}\end{array}$ \\
\hline 1 & 2.17 & 16.66 & 0.334 & 350 & & 20.09 & 0.512 & 273 \\
\hline 2 & 2.20 & 17.21 & 0.357 & 369 & 1.33 & 20.09 & 0.512 & 273 \\
\hline 3 & 2.19 & 17.40 & 0.357 & 363 & 1.26 & 20.17 & 0.542 & 272 \\
\hline 4 & 2.19 & 17.49 & 0.356 & 359 & 1.24 & 20.18 & 0.547 & 272 \\
\hline 5 & 2.19 & 17.54 & 0.354 & 356 & 1.24 & 20.18 & 0.548 & 272 \\
\hline 6 & 2.19 & 17.57 & 0.353 & 355 & 1.24 & 20.18 & 0.548 & 272 \\
\hline
\end{tabular}

${ }^{a}$ Shown are CIS/3-21G* results with $\mathbf{1}[\mathbf{8}]$. In each cycle, the Mulliken charges of the CT state from the previous cycle were used to generate the image charges. Image charges of the first cycle were generated from a calculation in a vacuum. ${ }^{b}$ Differences in vertical excitation energies. ${ }^{c}$ Differences in permanent dipole moments. ${ }^{d}$ Transition dipole moments. ${ }^{e}$ Electronic couplings.

3.2.2. Self-Consistency Iterations. The charges on a molecule polarize the solvent, and the reaction field in turn affects the electronic density of the molecule. Therefore, a rigorously correct solution should simultaneous satisfy both the Schrödinger equation and the Poisson-Boltzmann equation. If the exact solution is sufficiently close to the solution obtained in a vacuum, it is possible to start from the latter and obtain the former, through a series of iterations until self-consistency is achieved. For one of the molecules we studied, 1[8], we tested to see how different the self-consistent solution was from the one derived without self-consistency.

In Table 3, we listed results of iterating the calculation, where we used the Mulliken charges of the CT state from the previous cycle as the source charges of the ICA solvent model for calculation in the next cycle. For two different solvent polarities, it is shown that the solution converges quickly (coupling converges within six cycles in benzene or three cycles in acetonitrile). The final ET couplings through the self-consistent iteration are very similar to the values in the first cycle, where image charges derived from Mulliken charges obtained in a vacuum were used. Again, ET coupling depends mildly on the self-consistency of the ICA model.

3.2.3. Cavity Shapes. Cavity shape is a necessary setting in a dielectric solvent model. In the ICA model employed, a spherical cavity was assumed. There is an image charge solution
TABLE 4: Test of Electronic Coupling with a Rectangular Cavity in a Dielectric Solvent, with Molecule 1[8 $]^{a}$

\begin{tabular}{lcccccc}
\hline \multicolumn{1}{c}{ solvent } & $\epsilon$ & $d / \AA^{b}$ & $\Delta E / \mathrm{eV}$ & $\Delta \mu_{i} / \mathrm{au}$ & $\mu_{i f} / \mathrm{au}$ & $V / \mathrm{cm}^{-1}$ \\
\hline acetonitrile & & 3.0 & 0.486 & 20.46 & 1.34 & 255 \\
& 37.5 & 3.5 & 0.871 & 20.41 & 0.760 & 260 \\
& & 4.0 & 1.17 & 20.28 & 0.573 & 266 \\
ethyl acetate & 6.0 & 3.5 & 1.34 & 20.01 & 0.503 & 273 \\
tetrahydrofuran & 7.5 & 3.5 & 1.24 & 19.95 & 0.542 & 272 \\
dichloromethane & 9.1 & 3.5 & 1.17 & 20.28 & 0.574 & 266
\end{tabular}

${ }^{a}$ Shown are results from CIS/3-21G* calculations. The definition of symbols follow those of Table 3. ${ }^{b}$ When the center of nuclear charges of the molecule is set to origin, the two planar boundaries of the cavity in the $x(y, z)$ coordinate are placed at $\pm\left(d+\left|x_{\max }\right|\right)[ \pm(d+$ $\left.\left.\left|y_{\max }\right|\right), \pm\left(d+\left|z_{\max }\right|\right)\right]$, where $x_{\max }\left(y_{\max }, z_{\max }\right)$ is the largest atomic $x$ $(y, z)$ coordinate in their absolute values.

for rectangular cavities, and we tested to see if it could make a difference in the ET couplings.

For a solute placed in a rectangular box, there are infinite number of image charge sets due to successive mirror reflections. The image charges for each atom $\mathrm{A}$ are ${ }^{48}$

$$
q_{\mathrm{A} ; l, m, n}^{i m}=q^{\mathrm{A}}\left(\frac{1-\epsilon}{1+\epsilon}\right)^{|l|+|m|+|n|}
$$

where $-\infty<l, m, n<\infty$ but $l^{2}+m^{2}+n^{2} \neq 0$. The image charges are located at

$$
\begin{aligned}
\vec{r}_{\mathrm{A} ; l, m, n}^{i m}= & \left\{x,{ }_{\mathrm{A} ; i, j, k}^{i m}, y,{ }_{\mathrm{A} ; i, j, k}^{i m},,_{\mathrm{A} ; i, j, k}^{i m}\right\}= \\
& \left\{(-1)^{l} x_{\mathrm{A}}+l a,(-1)^{m} \mathrm{y}_{\mathrm{A}}+m b,(-1)^{n} z_{\mathrm{A}}+n c\right\}
\end{aligned}
$$

where $a, b$, and $c$ are the lengths of the rectangular box in the $x, y$, and $z$ direction, and $\vec{r}_{\mathrm{A}} \equiv\left\{x_{\mathrm{A}}, y_{\mathrm{A}}, z_{\mathrm{A}}\right\}$ are the Cartesian coordinates of the source charge A. As a first-order approximation, we included the closest six sets of image charges, located on the positive and negative direction of the Cartesian axis. In other words, image charges with $(l, m, n)=( \pm 1,0,0),(0, \pm 1$, $0)$, and $(0,0, \pm 1)$ are included. Results are listed in Table 4.

The solvation effect is stronger with this rectangular model, compared to the spherical model, because the second-order image charges, which offer the leading order correction, are always of the opposite signs to the first-order charges included (eq 8). Nevertheless, the results in Table 4 clearly indicate that the details of the solvation model do not significantly affect coupling.

With the results reported above, we conclude that a simple solvent model can be used for two purposes: (1) improving the quality of GMH coupling by lowering the energy of the CT state and (2) ensuring the quality of the calculation by varying the details of the solvent model, a test to see whether the Condon approximation is satisfied.

3.3. Norbornylogous Spacers Offer Through-Bond Superexchange Coupling. In Figure 1, the calculated ET coupling decays exponentially as the number of $\sigma$ bonds in the spacer is increased. Fitting our results to eq 2 we obtained a $\beta$ value of 0.99 bond $^{-1}\left(=0.90 \AA^{-1}\right)$ in acetonitrile with the $6-31 G^{*}$ basis set. This value agrees well with $\beta^{\prime}$ from the experimentally observed ET reaction rates defined as

$$
k_{\mathrm{ET}}=k_{\mathrm{ET}}^{0} \exp \left(-\beta^{\prime} d\right)
$$

which was found to be $0.92-1.25$ bond $^{-1}$, or $0.82-1.11 \AA^{-1}$, depending on the solvent. ${ }^{28,60,61}$

We tested for the role of spacers in mediating ET coupling by removing the norbornylogous bridge fragment. In Table 1 the couplings of these disconnected systems are reported in a 
separated row. The coupling values are much smaller and they decay very steeply compared to the other results. With these data, we confirm that ET coupling in this series is mediated by the through-bond mechanism.

3.3.1. Comparison with Previous Results. Larsson et al. ${ }^{57}$ and Clayton et al. ${ }^{59}$ have employed a semiempirically parametrized Hamiltonian with configuration interaction $(\mathrm{CNDO} / \mathrm{S} / \mathrm{CI})$ to calculate the transfer integral of the $\mathbf{1}[\boldsymbol{n}]$ system. Coupling values were derived from minimum energy differences between the $\mathrm{LE}\left(\mathrm{D}^{*} \mathrm{BA}\right)$ and $\mathrm{CT}\left(\mathrm{D}^{+} \mathrm{BA}^{-}\right)$states, with external charges ${ }^{57}$ or electric fields ${ }^{59}$ applied. Both results are very similar, ${ }^{62}$ and the coupling values are a few times smaller than the those inferred from experimental measurements.

The distance dependence $\beta$ values have been estimated in many previous works. The energy splitting of the two $\pi$ or $\pi^{*}$ orbitals in polynorbornane dienes have been measured and calculated via Koopmanns' theorem in the Hartree-Fock theory. $\beta$ 's were estimated to be 0.88 bond $^{-1}$ for hole transfer, and 1.2 bond $^{-1}$ for electron transfer. ${ }^{63}$ The coupling of the ground state and the CT state of $\mathbf{1}[\boldsymbol{n}]$ molecules was obtained from the charge-transfer absorption band ${ }^{64}$ and calculated with the extended Hückel Hamiltonian, ${ }^{65}$ with $\beta$ values 1.4 and $1.2 \AA^{-1}$ reported, respectively. The CNDO/S results mentioned above ${ }^{57,59}$ have a $\beta$ value of 1.38 bond $^{-1}$, which is slightly larger than that of experimental values $\left(0.92-1.25\right.$ bond $\left.^{-1}\right) .{ }^{28,60,61}$

Our ab initio ET couplings are larger than the values from $\mathrm{CNDO} / \mathrm{S}$, by at least a factor of 3 . The ab initio $\beta$ values are, in general, smaller than those derived from semiempirical Hamiltonians. Both the coupling magnitudes and the $\beta$ value we obtained are much closer to those derived from experimental data $^{58}$ than those previously reported. Such results may imply an excellent predictive capability of first-principle based computation.

Semiempirical methods simplify the Hamiltonian in two aspects, one is omitting certain two-electron integrals, and the other is parametrizing the one and two-electron integrals. In CNDO, many two electron integrals are neglected, including many Coulomb terms containing differential overlaps and all of the exchange integrals. The orbital shapes (how they decay in space) are implicitly assumed in parametrized integrals. Although at present we are not sure of the exact source of the discrepancy between the CNDO/S values and our ab initio results, we note that both types of simplifications in CNDO are potentially able to contribute to the discrepancy observed.

3.4. Cofacial ET Systems: Through-Space Coupling. Another system we studied is shown in Chart 2. The donor (ANI) was placed either in a $\pi$-stacked arrangement with an acceptor fragment (2a, the 1,5 substituted molecule) or at the opposite ends of the rigid spacer ( $\mathbf{2} \mathbf{b}$, the 1,8 substituted one) ${ }^{29}$ In Table 5 we listed our calculated results. Again, good agreement between calculated data and experimentally inferred values is seen. In this case, the use of the ICA solvation model, with a spherical cavity, does not significantly affect the outcome, again indicating that the CT character is within the Condon approximation region.

We have also calculated the through-space coupling from disconnected donor/acceptor molecules without the bridge, and the results are very similar to those of full molecules (Table 5). This result supports a through-space coupling mechanism and is consistent with previous conclusions. The use of a larger basis set or inclusion of diffusive basis functions has a larger effect in the final coupling values than in the $\mathbf{1}[\boldsymbol{n}]$ system. This is another characteristic of through-space coupling. Our results indicate that CIS-GMH is able to treat the electron tunneling
TABLE 5: Electronic Coupling $\left(\mathrm{cm}^{-1}\right)$ of ANI*-DBO-PI $\rightarrow$ ANI $^{+}$-DBO-PI

\begin{tabular}{|c|c|c|}
\hline system & solvent & $V / \mathrm{cm}^{-1 a}$ \\
\hline \multicolumn{3}{|c|}{ 1,5-Substituted ANI-DBO-PI (2a) } \\
\hline \multirow[t]{3}{*}{ full molecule } & vacuum & $215 / 252^{b} / 241^{c}$ \\
\hline & benzene & 194 \\
\hline & acetonitrile & 192 \\
\hline $\begin{array}{l}\text { without DBO fragment } \\
\text { experiments }^{d}\end{array}$ & vacuum & $\begin{array}{l}185 / 242^{b} / 216^{c} \\
207\end{array}$ \\
\hline \multicolumn{3}{|c|}{ 1,8-Substituted ANI-DBO-PI (2b) } \\
\hline \multirow[t]{3}{*}{ full molecule } & vacuum & $1.84 / 2.37^{b} / 3.96^{c}$ \\
\hline & benzene & 1.72 \\
\hline & acetonitrile & 1.48 \\
\hline \multirow{2}{*}{$\begin{array}{l}\text { without DBO fragment } \\
\text { experiments }^{d}\end{array}$} & vacuum & $0^{e} / 0.096^{b} / 0^{c, e}$ \\
\hline & & 0 \\
\hline
\end{tabular}

${ }^{a}$ Obtained with $3-21 \mathrm{G}^{*}$ basis set unless otherwise noted. ${ }^{b} 3-21+\mathrm{G}^{*}$ basis set. ${ }^{c} 6-31 \mathrm{G}^{*}$ basis set. ${ }^{d}$ Reference $29 .{ }^{e}$ Coupling is smaller than $0.005 \mathrm{~cm}^{-1}$.

through a vacuum, and this implies that the wave functions in the asymptotic region are treated properly.

3.5. Scheme for Both Though-Bond and Through-Space Coupling. In the previous sections, it is shown that our scheme using CIS-GMH with an ICA solvation model can properly estimate both through-bond and through-space coupling. The underlying interactions of the two contributions, as indicated in eq 7, are different in nature. Interactions between the excited electron (typically in a $\pi^{*}$ orbital in many photoinduced systems) and electrons in bridge $\sigma$ orbitals must be treated properly to obtain correct bridge-mediated coupling values. Likewise, in the through-space case, the wave function's exponential extension in space needs to be properly reproduced in the calculation for a good estimate. We show that the CIS-GMH scheme is able to treat systems dominated by either through-bond (superexchange) or through-space contributions, with results very similar to those derived experimentally, indicating a good capability of modeling both types of interactions in ET couplings. With this result, we conclude that CIS-GMH is a useful scheme to characterize couplings for photoinduced ET reactions.

The GMH formalism has been generalized to three-state situation. ${ }^{66}$ This approach will be a suitable alternative solution to resolve the coupling of the CT state to other high-lying LE states. From the configurations listed in Table 2, it seems necessary to include two or more LE states in the multistate GMH approach. Manual assignment and inclusion of important states will be necessary. It would be interesting to see how results from our solvent stabilization data compared with results derived from the multi-state GMH scheme.

\section{Concluding Remarks}

For photoinduced ET systems, we have developed an ab initio scheme to properly account for the ET coupling magnitudes. We showed that CIS can be used for the excited states and CT states, and that the coupling can be estimated via the GMH method. For the cases where CT states are erroneously coupled to high lying excited states, ICA can model the effect of a polar solvent and reduce the CT state energy. With the ICA model, we showed that the ET coupling of a LE state to the CT state (which is another excited state) can be calculated with a CISGMH scheme. Using the Condon approximation, the reliability of the final coupling values was ensured by the insensitive response to many details of the solvent model, including the solvent polarity, shape and size of the cavity and extent of selfconsistency between quantum mechanics and classical electro- 
statics. We demonstrated that our CIS-GMH scheme is able to characterize both through-bond and through-space ET coupling, with results close to experimentally derived values. Thus, CISGMH, with a solvent model when necessary, can offer good accounts of photoinduced ET couplings.

Acknowledgment. We gratefully acknowledge the Nano research grants from Academia Sinica. H.C.C. acknowledges support from the National Science Council, Republic of China (Grant No. 93-2113-M-001-012-).

Supporting Information Available: Structure, bond lengths, bond angles and couplings of $\mathbf{1}[\mathbf{8}]$. This material is available free of charge via the Internet at http://pubs.acs.org.

\section{References and Notes}

(1) Marcus, R. A.; Sutin, N. Biochim. Biophys. Acta 1985, 811, 265

(2) Balzani, V., Ed.; Electron Transfer in Chemistry; Wiley-VCH: Weinheim, 2001;Vols. 1-5.

(3) Jortner, J., Bixon, M., Eds. Electron Transfer-From Isolated Molecules to Biomolecules; Wiley-Interscience: New York, 1999; Parts 1 and 2.

(4) Barbara, P. F.; Meyer, T. J.; Ratner, M. A. J. Phys. Chem. A 1996, 100,13148 .

(5) Wasielewski, M. R. Chem. Rev. 1992, 92, 435

(6) Moser, C. C.; Keske, J. M.; Warncke, K.; Farid, R. S.; Dutton, P. L. Nature 1992, 355, 796.

(7) Gray, H. B.; Winkler, J. R. Annu. Rev. Biochem. 1996, 65, 537.

(8) Gust, D.; Moore, T.; Moore, A. Acc. Chem. Res. 2001, 34, 40.

(9) Avriam, A.; Ratner, M. A. Chem. Phvs. Lett. 1974, 29, 277.

(10) Metzger, R. M. Chem. Rev. 2003, 103, 3803.

(11) de Silva, A. P.; Gunaratne, H. Q. N.; Gunnlaugsson, T.; Huxley, A. J. M.; McCoy, C. P.; Rademacher, J. T.; Rice, T. E. Chem. Rev. 1997, 97, 1515.

(12) Davis, W. B.; Svec, W. A.; Ratner, M. A.; Wasielewski, M. R. Nature 1998, 396, 60.

(13) Ballardini, R.; Balzani, V.; Credi, A.; Gandolfi, M. T.; Venturi, M. Acc. Chem. Res. 2001, 34, 445.

(14) Guldi, D. Chem. Soc. Rev. 2002, 31, 22

(15) Imahori, H.; Mori, Y.; Matano, Y. J. Photochem. Photobiol. C 2003, 4,51 .

(16) Kanis, D. R.; Ratner, M. A.; Marks, T. J. Chem. Rev. 1994, 94, 195.

(17) Bella, S. D. Chem. Soc. Rev. 2001, 30, 355.

(18) Marcus, R. A. J. Chem. Phvs. 1965, 43, 679-701.

(19) Hoffmann, R. Acc. Chem. Res. 1971, 4, 1.

(20) Clayton, A. H. A.; Scholes, G. D.; Ghiggino, K. P.; Paddon-Row,

M. N. J.Phvs. Chem. 1996, 100, 10912.

(21) McConnell, H. M. J. Chem. Phvs. 1961, 35, 508.

(22) Herzfeld, K. F. J. Chem. Phys. 1942, 10, 508

(23) Hsu, C. P.; Marcus, R. A. J. Chem. Phvs. 1997, 106, 584.

(24) Paddon-Row, M. N.; Jordon, K. D. In Modern Models of Bonding and Delocalization; Liebman, J. F.; Greenberg, A., Eds.; VCH Publishers: New York, 1988; Vol. 6 p 115

(25) Closs, G. L.; Calcaterra, L. T.; Green, N. J.; Penfield, K. W.; Miller, J. R. J. Phys. Chem. 1986, 90, 3673.

(26) Closs, G. L.; Miller, J. R. Science 1988, 240, 440.

(27) Shephard, M. J.; Paddon-Row, M. N.; Jordan, K. D. J. Am. Chem. Soc. 1994, 116, 5328.

(28) Oevering, H.; Paddon-Row, M. N.; Heppener, H.; Oliver, A. M.; Cotaaris, E.; Verhoeven, J. W.; Hush, N. S. J. Am. Chem. Soc. 1987, 109, 3258.
(29) Gosztola, D.; Wasielewski, B. W. M. J. J. Photochem. Photobiol. A 1996, 102, 71.

(30) Weiss, E. A.; Ahrens, M. J.; Sinks, L. E.; Gusev, A. V.; Ratner, M. A.; Wasielewski, M. R. J. Am. Chem. Soc. 2004, 126, 5577.

(31) Cave, R. J.; Newton, M. D. Chem. Phvs. Lett. 1996, 249, 15

(32) Cave, R. J.; Newton, M. D.; Kumar, K.; Zimmt, M. B. J. Phvs. Chem. 1995, 99, 17501.

(33) Kumar, K.; Kurnikov, I. V.; Beratan, D. N.; Waldeck, D. H.; Zimmt, M. B. J. Phys. Chem. A 1998, 102, 5529 .

(34) Anderson, W. P.; Cundari, T. R.; Zerner, M. C. Int. J. Ouantum. Chem. 1991, 39, 31.

(35) Ohta, K.; Closs, G. L.; Morokuma, K.; Green, N. J. J. Am. Chem. Soc. 1986, 108, 1319 .

(36) Broo, A.; Larsson, S. Chem. Phys. 1990, 148, 103.

(37) Farazdel, A.; Dupuis, M.; Clementi, E.; Aviram, A. J. Am. Chem. Soc. 1990, 112, 4206-4214.

(38) Koopmans, T. Physica 1934, 1, 104-113.

(39) Newton, M. D. Chem. Rev. 1991, 91, 767.

(40) You, Z.-Q.; Shao, Y.; Hsu, C.-P. Chem. Phvs. Lett. 2004, 390, 116.

(41) Cave, R. J.; Newton, M. D. J. Chem. Phvs. 1997, 106, 9213.

(42) Toutounji, M. M.; Ratner, M. A. J. Phvs. Chem. A 2000, 104, 8566. 4154 .

(44) Dreuw, A.; Head-Gordon, M. J. Am. Chem. Soc. 2004, 126, 4007.

(45) Tomasi, J.; Persico, M. Chem. Rev. 1994, 94, 2027.

(46) Hasegawa, J.; Nakatsuji, H. J. Phvs. Chem. B 1998, 102, 10420

(47) Friedman, H. L. Mol. Phys 1975, 29, 1533. 2973.

(48) Yang, P. K.; Liaw, S. H.; Lim, C. J. Phvs. Chem. B 2002, 106,

(49) Condon, E. U. Phvs. Rev. 1928, 32, 858.

(50) Löwdin, P. O. J. Math. Phvs. 1962, 3, 969.

(51) Löwdin, P. O. J. Mol. Spectrosc. 1963, 10, 12.

(52) Larsson, S. J. J. Am. Chem. Soc. 1981, 103, 4034.

(53) Becke, A. D. J. Chem. Phys. 1993, 98, 6548.

(54) The composed structure is diabatic in nature, with the donor attached to a small bridge optimized in its excited state. In the Supporting Information, we show that the diabatic structure is very close to the adiabatic (fully relaxed) one. The calculation performed in this manner is in the reactant state geometry, where the many properties of the nuclear diabatic and adiabatic states are similar. In all calculations the electronic adiabatic state was obtained, as required by the GMH scheme. We note that the use of the diabatic structure has little impact on the electronic state.

(55) Kong, J.; et al. J. Comput. Chem. 2000, 21, 1532.

(56) Kemp, M.; Mujica, V.; Ratner, M. A. J. Chem. Phys. 1994, 101, 5172 .

(57) Larsson, S.; Volosov, A. J. Chem. Phys. 1986, 85, 2548.

(58) Bixon, M.; Jortner, J.; Verhoeven, J. W. J. Am. Chem. Soc. 1994, 116,7349 .

(59) Clayton, A.; Ghiggino, K. P.; Wilson, G. J.; Paddon-Row, M. N. J. Phvs. Chem. 1993, 97, 7962.

(60) Paddon-Row, M. N.; Oliver, A. M.; Warman, J. M.; Smit, K. J.; de Haas, M. P.; Oevering, H.; Verhoeven, J. W. J. Phys. Chem. 1988, 92, 6958.

(61) Oliver, A. M.; Craig, D.; Paddon-Row, M. N.; Kroon, J.; Verhoeven, J. W. Chem. Phvs. Lett. 1988, 150, 366.

(62) We have assumed that $\Delta$ 's reported in ref 57 are the coupling magnitude $V$.

(63) Jordan, K. D.; Paddon-Row, M. N. Chem. Rev. 1992, 92, 395.

(64) Penfield, K. W.; Miller, J. R.; Paddon-Row, M. N.; Cotsaris, E.; Oliver, A. M.; Hush, N. S. J. Am. Chem. Soc. 1987, 109, 5061

(65) Siddarth, P.; Marcus, R. A. J. Phys. Chem. 1990, 94, 3213

(66) Rust, M.; Lappe, J.; Cave, R. J. J. Phvs. Chem. A 2002, 106, 3930. 